\title{
Timed Control with Observation Based and Stuttering Invariant Strategies
}

\author{
Franck Cassez ${ }^{1, \star \star \star \star}$, Alexandre David ${ }^{2}$, Kim G. Larsen ${ }^{2}$, Didier Lime ${ }^{1, \star}$, \\ Jean-François Raskin ${ }^{3, \star \star \star}$ \\ 1 IRCCyN, CNRS, Nantes, France \\ \{franck. cassez, didier. lime\}@irccyn.ec-nantes.fr \\ 2 CISS, CS, Aalborg University, Denmark \\ \{adavid,kgl\}@cs.aau.dk \\ ${ }^{3}$ Computer Science Department, Université Libre de Bruxelles (U.L.B.), Belgium \\ jraskin@ulb.ac.be
}

\begin{abstract}
In this paper we consider the problem of controller synthesis for timed games under imperfect information. Novel to our approach is the requirements to strategies: they should be based on a finite collection of observations and must be stuttering invariant in the sense that repeated identical observations will not change the strategy. We provide a constructive transformation to equivalent finite games with perfect information, giving decidability as well as allowing for an efficient on-the-fly forward algorithm. We report on application of an initial experimental implementation.
\end{abstract}

\section{Introduction}

Timed automata introduced by Alur and Dill [2] is by now a well-established formalism for representing the behaviour of real-time systems. Since their definition several contributions have been made towards the theoretical and algorithmic characterization of this formalism. In particular, industrial mature tools supporting model-checking for timed automata now exist $[7,6]$.

More recently the problem of controller synthesis for timed automata based models have been considered: i.e. given a timed game automaton modelling the possible moves of an environment as well as the possible moves of a control program, the problem consists in synthesizing a strategy for the control program in order that a given control objective is met no matter how the environment behaves [15].

* Work supported by the French National Research Agency ANR-06-SETI-DOTS.

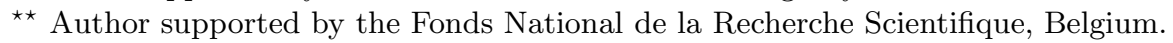

$\star \star \star *$ Author supported by the Belgian FNRS grant 2.4530.02 of the FRFC project "Centre Fédéré en Vérification" and by the project "MoVES", an Interuniversity Attraction Poles Programme of the Belgian Federal Government. 
Controller synthesis and time-optimal controller synthesis for timed games was shown decidable in [4] and [3]. First steps towards efficient synthesis algorithms were taken in $[1,16]$. In [9] a truly on-the-fly algorithm based on a mixture of forward search and backwards propagation was proposed and later used as the basis for an efficient implemention in the tool UpPaAl Tiga [5].

In all of the papers cited above it has been assumed that the controller has perfect information about the plant: at any time, the controller knows precisely in what state the environment is. In general however - e.g. due to limited sensors - a controller will only have imperfect (or partial) information about the state of the environment. In the discrete case it is well known how to handle partial observability, however for the timed case it has been shown in [8] that the controller synthesis problem under partial observability is in general undecidable. Fixing the resources of the controller (i.e. a maximum number of clocks and maximum allowed constants in guards) regains decidability [8], a result which also follows from the quotient and model construction results of $[12,13]$.

In this paper we also deal with the problem of controller synthesis for timed games under imperfect information following the approach of $[10,17]$. That is, the imperfect information is given in terms of (a finite number of possible) observations to be made on the system configurations, providing the sole basis for the strategy of the controller. However, in contrast to $[10,17]$, which is essentially turn-based in the untimed setting, we will here consider a more general framework, where in each step the controller and environment are competing. In particular, the strategy of the controller is supposed to be stuttering invariant, i.e. the strategy will not be affected by a sequence of environment or time steps unless changes in the observations occur.

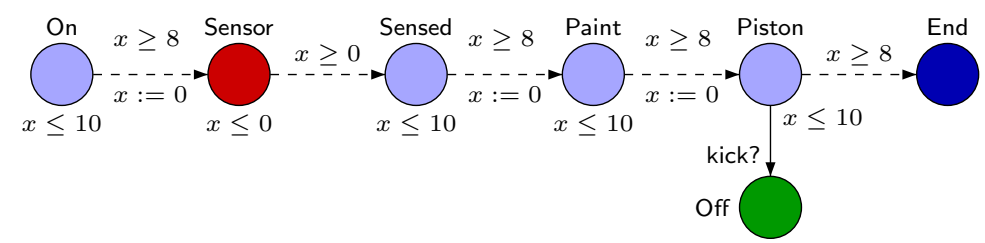

Fig. 1. Timed Game with Imperfect Information.

To illustrate the concepts of imperfect information and stuttering invariance consider the timed game automaton in Figure 1 modelling a production system for painting a box moving on a conveyor belt. The various locations indicate the position of the box in the system: in Sensor a sensor is assumed to reveal the presence of the box, in Sensed the box is moving along the belt towards the painting area, in Paint the actual painting of the box takes place, in Piston the box may be kick?'ed off the belt leading to Off; if the box is not kicked off it ends in End. All phases are assumed to last between 8 and 10 seconds, except for the phase Sensor, which is instantaneous. The uncontrollability of this timing uncertainty is indicated by the dashed transitions between phases. The controller should now issue a single kick?'command at the appropriate moment in order 
to guarantee that the box will - regardless of the above timing uncertainy be kicked off the belt. However the controller has imperfect information of the position of the box in the system. In particular, the controller cannot directly observe whether the box is in the Sensed, Paint or in the Piston phase nor can the value of the clock $x$ be observed. Still equipping the controller with its own clock $y$-which it may reset and test (against a finite number of predicates) it might be possible to synthesize a control strategy despite having only partial information: in fact it may be deduced that the box will definitely be in the Piston area within 20-24 seconds after being sensed. In contrast, an increased timing uncertainty where a phase may last between 6 and 10 seconds will make a single-kick? strategy impossible.

The main contributions of this paper are: $(i)$ we show how a variant of the subset construction of $[10,17]$ allows us to transform a timed game $H$ with imperfect information into an equivalent game $G(H)$ of perfect information; (ii) we show that $G(H)$ can be effectively and symbolically computed and this implies that the control problem under imperfect information is decidable; this allows us to apply the efficient on-the-fly forward algorithm from [9] and (iii) we report on application of an initial experimental implementation of this algorithm and a number of heuristics for minimizing the explored state-space as well as the size of the finally synthesized strategy.

The detailed proofs can be found in the extended version available from the authors web pages.

\section{Timed Games and Observation Based Strategies}

In this section, we define the timed game structures, the notion of strategies that we are interested in, and useful vocabulary for the rest of the paper. We denote $\mathbb{R}_{\geq 0}$ the set of non-negative reals and $\mathbb{R}_{>0}=\mathbb{R}_{\geq 0} \backslash\{0\}$ and $A^{B}$ the set of mappings from $B$ to $A$.

Timed game structures (TGS) will be defined using a timed automaton like notation. The semantics of the notation will be defined by a two-player labeled timed transition system (2-LTTS), and the games will be played on this 2-LTTS.

Definition 1 (2-LTSS). A 2-player labeled timed transition system (2-LTTS) is a tuple $\left(S, s_{0}, \Sigma_{1}, \Sigma_{2}, \rightarrow\right)$ where $S$ is a (infinite) set of states, $s_{0}$ is the initial state, $\Sigma_{1}$ and $\Sigma_{2}$ are the two disjoint alphabets of actions for Player 1 and Player 2 respectively, and $\rightarrow \subseteq S \times \Sigma_{1} \cup \Sigma_{2} \cup \mathbb{R}_{>0} \times S$ is the transition relation.

Given a state $s \in S$, we define enable(s) as the set of $\sigma \in \Sigma_{1} \cup \Sigma_{2} \cup \mathbb{R}_{>0}$ such that there exists $s^{\prime}$ and $\left(s, \sigma, s^{\prime}\right) \in \rightarrow$.

Let $X$ be a finite set of real-valued variables called clocks. Let $M$ be a natural number. We note $\mathcal{C}(X, M)$ the set of constraints $\varphi$ generated by the grammar: $\varphi::=x \sim k|x-y \sim k| \varphi \wedge \varphi$ where $k \in \mathbb{Z} \cap[0, M], x, y \in X$ and $\sim \in$ $\{<, \leq,=,>, \geq\} \cdot \mathcal{B}(X, M)$ is the subset of $\mathcal{C}(X, M)$ generated by the following grammar: $\varphi::=\top\left|k_{1} \leq x<k_{2}\right| \varphi \wedge \varphi$ where $k, k_{1}, k_{2} \in \mathbb{Z} \cap[0, M], k_{1}<k_{2}$, $x \in X$, and $\top$ is the boolean constant true. In the sequel, we will restrict our 
attention to bounded timed automata where clock values are all bounded by a natural number $M$; this does not reduce the expressive power of timed automata. Given a natural number $M$, an $M$-valuation of the variables in $X$ is a mapping $X \mapsto \mathbb{R}_{\geq 0} \cap[0, M]$. We also use the notation $[X \rightarrow[0, M]]$ for valuations and $\mathbf{0}$ for the valuation that assigns 0 to each clock. For $Y \subseteq X$, we denote by $v[Y]$ the valuation assigning 0 (resp. $v(x)$ ) for any $x \in Y$ (resp. $x \in X \backslash Y$ ). Let $t \in \mathbb{R}_{\geq 0}$, $v$ be an $M$-valuation for the set of clocks $X$, if for all $x \in X, v(x)+t \leq M$ then $v+t$ is the $M$-valuation defined by $(v+t)(x)=v(x)+t$ for all $x \in X$. For $g \in \mathcal{C}(X, M)$ and $v \in\left(\mathbb{R}_{\geq 0} \cap[0, M]\right)^{X}$, we write $v \models g$ if $v$ satisfies $g$ and $\llbracket g \rrbracket$ denotes the set of valuations $\left\{v \in\left(\mathbb{R}_{\geq 0} \cap[0, M]\right)^{X} \mid v \models g\right\}$. An $M$-zone $Z$ is a subset of $\left(\mathbb{R}_{\geq 0} \cap[0, M]\right)^{X}$ s.t. $Z=\llbracket g \rrbracket$ for some $g \in \mathcal{C}(X, M)$.

Definition 2 (Timed Game Structure). Let $M$ be a natural number, an $M$ timed game structure $(M-T G S)$ is a tuple $H=\left(L, \iota, X, \delta, \Sigma_{1}, \Sigma_{2}\right.$, inv, $\left.\mathcal{P}\right)$ where:

- L is a finite set of locations,

$-\iota \in L$ is the initial location,

- $X$ is a finite set of real-valued clocks,

- $\Sigma_{1}, \Sigma_{2}$ are two disjoint alphabets of actions, $\Sigma_{1}$ is the set of actions of Player 1 and $\Sigma_{2}$ the set of actions of Player 2,

$-\delta \subseteq\left(L \times \mathcal{B}(X, M) \times \Sigma_{1} \times 2^{X} \times L\right) \cup\left(L \times \mathcal{C}(X, M) \times \Sigma_{2} \times 2^{X} \times L\right)$ is partitioned into transitions ${ }^{1}$ of Player 1 and transitions of Player 2.

- inv : $L \rightarrow \mathcal{B}(X, M)$ associates to each location its invariant.

- $\mathcal{P}$ is a finite set of pairs $(K, \varphi)$ where $K \subseteq L$ and $\varphi \in \mathcal{B}(X, M)$, called observable predicates.

In the definition above, each observable predicate $(K, \varphi) \in \mathcal{P}$ is a predicate over the state space of the TGS, i.e. the set $L \times[X \rightarrow[0, M]]$. For $l \in L$ and $v$ an $M$-valuation of the clocks in $X$, we write $(l, v) \models(K, \varphi)$ iff $l \in K$ and $v \models \varphi$. Two pairs $\left(l_{1}, v_{1}\right),\left(l_{2}, v_{2}\right)$ that satisfy the same observable predicates from $\mathcal{P}$ have the same observation (they can not be distinguished by our controllers). So, an observation is a function $o: \mathcal{P} \rightarrow\{0,1\}$, or equivalently, a class of equivalent states w.r.t $\mathcal{P}$. We note $\mathcal{O}$ the set of functions $[\mathcal{P} \rightarrow\{0,1\}]$, it is called the set of observations of the system. With each TGS $H$ with set of observable predicates $\mathcal{P}$, we associate the function $\gamma$ that maps observations to classes of equivalent states, i.e. $\gamma: \mathcal{O} \rightarrow 2^{L \times[X \rightarrow[0, M]]}$, and it is defined as follows:

$$
\gamma(o)=\left\{(l, v) \mid \bigwedge_{(K, \varphi) \mid o(K, \varphi)=1}(l, v) \models(K, \varphi) \wedge \bigwedge_{(K, \varphi) \mid o(K, \varphi)=0}(l, v) \forall(K, \varphi)\right\}
$$

Note that the set of observations $\mathcal{O}$ defines a partition of the state space of the $M$-TGS, i.e. $\bigcup_{o \in \mathcal{O}} \gamma(o)=L \times[X \rightarrow[0, M]]$, and for all $o_{1}, o_{2} \in \mathcal{O}$, if $o_{1} \neq o_{2}$, then $\gamma\left(o_{1}\right) \cap \gamma\left(o_{2}\right)=\emptyset$. Given $(l, v)$ we write $\gamma^{-1}(l, v)$ for the observation $o$ of

\footnotetext{
${ }^{1}$ Note that we impose that guards of Player 1's transitions are left closed. This ensures that, when a guard becomes true for an action owned by Player 1, there is always a first instant where it becomes true.
} 
state $(l, v)$, i.e. $\gamma^{-1}(l, v)$ is the function $o: \mathcal{P} \rightarrow\{0,1\}$ s.t. $\forall p \in \mathcal{P}, o(p)=1 \Longleftrightarrow$ $(l, v) \models p$.

We associate with any $M$-TGS $H$ a semantics in the form of a (infinite state) 2-LTTS. The state space of the 2-LTTS will be composed of elements of the form $(l, v)$ where $l$ is a location of the TGS and $v$ is a valuation of the clocks. In order to avoid deadlocks in the 2-LTTS, we require that our TGS are deadlock-free ${ }^{2}$, that is, for every state $(l, v)$ such that $v \models \operatorname{inv}(l)$, there exists $\sigma \in \Sigma_{2} \cup \mathbb{R}_{>0}$, such that either there is a transition $\left(l, g, \sigma, Y, l^{\prime}\right) \in \delta$ such that $v \models g$ and $v[Y] \models \operatorname{inv}\left(l^{\prime}\right)$, or for all $t^{\prime}, 0 \leq t^{\prime} \leq \sigma, v+t^{\prime} \models \operatorname{inv}(l)$.

Definition 3 (Semantics of a TGS). The semantics of an $M-T G S H=$ $\left(L, \iota, X, \delta, \Sigma_{1}, \Sigma_{2}\right.$, inv, $\left.\mathcal{P}\right)$ is a 2 -LTTS $S_{H}=\left(S, s_{0}, \Sigma_{1}, \Sigma_{2}, \rightarrow\right)$ where:

$-S=\left\{(l, v) \mid l \in L \wedge v \in\left(\mathbb{R}_{\geq 0} \cap[0, M]\right)^{X} \wedge v \models \operatorname{inv}(l)\right\} ;$

$-s_{0}=(\iota, \mathbf{0})$;

- the transition relation is composed of

(i) discrete transitions. For all $\left(l_{1}, v_{1}\right),\left(l_{2}, v_{2}\right) \in S$, for all $\sigma \in \Sigma_{1} \cup \Sigma_{2}$, $\left(\left(l_{1}, v_{1}\right), \sigma,\left(l_{2}, v_{2}\right)\right) \in \rightarrow$ iff there exists a transition $\left(\ell, g, a, Y, \ell^{\prime}\right) \in \delta$ such that $\ell=l_{1}, \ell^{\prime}=l_{2}, v_{1} \models g$, and $v_{2}=v_{1}[Y]$;

(ii) time transitions. For all $\left(l_{1}, v_{1}\right),\left(l_{2}, v_{2}\right) \in S$, for all $t \in \mathbb{R}_{>0}$, there is a transition $\left(\left(l_{1}, v_{1}\right), t,\left(l_{2}, v_{2}\right)\right) \in \rightarrow$ iff $l_{1}=l_{2}, v_{2}=v_{1}+t$, and for all $t^{\prime}$, $0 \leq t^{\prime}<t,\left(l_{1}, v_{1}+t^{\prime}\right) \in S$ and $\gamma^{-1}\left(l_{1}, v_{1}+t^{\prime}\right)=\gamma^{-1}\left(l_{1}, v_{1}\right)$.

Remark 1. This semantics has the following important property: changes of observations can occur only during a discrete transition, or at the last point of a time delay. This is consistent with our definition of observations using constraints in $\mathcal{B}(X, M)$ : the form of the constraints implies that either for all $t \geq 0$, $(l, v) \stackrel{t}{\rightarrow}(l, v+t)$, and $\gamma^{-1}(l, v+t)=\gamma^{-1}(l, v)$, or there is a first instant $t_{0}>0$ s.t. $(l, v) \stackrel{t_{0}}{\longrightarrow}\left(l, v+t_{0}\right)$ and $\gamma^{-1}\left(l, v+t_{0}\right) \neq \gamma^{-1}(l, v)$, and for all $0 \leq t^{\prime}<t_{0}$, $\gamma^{-1}\left(l, v+t^{\prime}\right)=\gamma^{-1}(l, v)$.

The 2-LTTS of a TGS has no deadlock because a TGS is deadlock-free. This also implies that any state of the 2-LTTS is the source of an infinite path. As a TGS is bounded, these infinite paths contain infinitely many discrete steps and in the sequel we will consider only these type of infinite paths.

Playing with Observation Based Stuttering Invariant Strategies. In the remainder of this section, we will define the rules of the timed games that we want to consider. We start by an informal presentation and then turn to the formalization.

Player 1 and Player 2 play on the underlying 2-LTTS of a TGS as follows. Player 1 has to play according to observation based stuttering invariant strategies (OBSI strategies for short). Initially and whenever the current observation of the system state changes, Player 1 either proposes an action $\sigma_{1} \in \Sigma_{1}$, or the special

\footnotetext{
${ }^{2}$ And more precisely, either time can elapse or Player 2 can do a discrete action from any state: thus Player 1 cannot block the game by refusing to take its actions.
} 
action delay. When Player 1 proposes $\sigma_{1}$, this intuitively means that he wants to play the action $\sigma_{1}$ whenever this action is enabled in the system. When Player 1 proposes delay, this means that he does not want to play discrete actions until the next change of observation, he is simply waiting for the next observation. Thus, in the two cases, Player 1 sticks to his choice until the observation of the system changes: in this sense he is playing with an observation based stuttering invariant strategy. Once Player 1 has committed to a choice, Player 2 decides of the evolution of the system until the next observation but respects the following rules:

1. if the choice of Player 1 is a discrete action $\sigma_{1} \in \Sigma_{1}$ then Player 2 can choose to play, as long as the observation does not change, either $(i)$ a discrete actions in $\Sigma_{2} \cup\left\{\sigma_{1}\right\}$ or $(i i)$ let time elapse as long as $\sigma_{1}$ is not enabled. This entails that $\sigma_{1}$ is urgent,

2. if the choice of Player 1 is the special action delay then Player 2 can choose to play, as long as the observation does not change, any of its discrete actions in $\Sigma_{2}$ or let time pass,

3. the turn is back to Player 1 as soon as the next observation is reached.

Plays. In the following, we define plays of a game where choices of Player 1 are explicitly mentioned. A play in $H$ is an infinite sequence of transitions in $S_{H}, \rho^{H}=\left(l_{0}, v_{0}\right) c_{0} \sigma_{0}\left(l_{1}, v_{1}\right) c_{1} \sigma_{1} \ldots\left(l_{n}, v_{n}\right) c_{n} \sigma_{n} \ldots$, such that for all $i \geq 0$, $\left(l_{i}, v_{i}\right) \stackrel{\sigma_{i}}{\longrightarrow}\left(l_{i+1}, v_{i+1}\right)$ and

- either $\sigma_{i} \in\left\{c_{i}\right\} \cup \Sigma_{2}$, or

$-\sigma_{i} \in \mathbb{R}_{>0}^{X}$ and $\forall 0 \leq t<\sigma_{i}, c_{i} \notin$ enable $\left(l_{i}, v_{i}+t\right)$ (time elapses only when the choice of Player 1 is not enabled). ${ }^{3}$

- if $\sigma_{i}$ and $\sigma_{i+1}$ are in $\mathbb{R}_{>0}^{X}$ then $\gamma^{-1}\left(l_{i}, v_{i}\right) \neq \gamma^{-1}\left(l_{i+1}, v_{i+1}\right)$.

We write Play $((l, v), H)$ for the set of plays in $H$ that start at state $(l, v)$. We write Play $(H)$ for the initial plays that start at the initial state of $H$, that is the set Play $((\iota, \mathbf{0}), H)$.

Prefixes, Strategies, and Outcomes. A prefix of $H$ is a prefix of a play in $H$ that ends in a state of $H$. We note $\operatorname{Pref}((l, v), H)$ for the set of prefixes of plays in $H$ that starts in $(l, v)$, i.e. plays in $\operatorname{Play}((l, v), H)$. We note $\operatorname{Pref}(H)$, for prefixes of initial plays in $H$, i.e. prefixes of plays in Play $(H)$. Let $\rho^{H}=$ $\left(l_{0}, v_{0}\right) c_{0} \sigma_{0} \cdots\left(l_{n}, v_{n}\right) c_{n} \sigma_{n} \cdots$ be a play or a prefix of a play, $\rho^{H}(n)$ denotes the prefix up to $\left(l_{n}, v_{n}\right)$. In the sequel, we measure the length of a prefix by counting the number of states that appear in the prefix. For example, $\rho^{H}(n)$ has a length equal to $n+1$. A strategy for Player 1 in $H$ is a function $\lambda^{H}$ : $\operatorname{Pref}(H) \rightarrow \Sigma_{1} \cup\left\{\right.$ delay\}. The outcome of a strategy $\lambda^{H}$ in $H$ is the set of plays $\rho^{H}=\left(l_{0}, v_{0}\right) c_{0} \sigma_{0}\left(l_{1}, v_{1}\right) c_{1} \sigma_{1} \ldots\left(l_{n}, v_{n}\right) c_{n} \sigma_{n} \ldots$ such that $l_{0}=\iota, v_{0}=\mathbf{0}$ and for all $i \geq 0, c_{i}=\lambda^{H}\left(\rho^{H}(i)\right)$. We note Outcome ${ }^{H}\left(\lambda^{H}\right)$ this set of plays.

\footnotetext{
${ }^{3}$ Remember that delay is never enabled and if Player 1 wants to let time elapse he plays delay.
} 
Consistent Plays, Choice Points and OBSI Strategies in H. We are interested in strategies for Player 1 where the choice of action can only change if the observation of the state of the system changes. Such a strategy is called an $o b-$ servation based stuttering invariant strategy as presented before. When Player 1 plays such a strategy, the resulting plays have the property of being consistent. This notion is defined as follows. A play $\rho^{H}=\left(l_{0}, v_{0}\right) c_{0} \sigma_{0} \cdots\left(l_{n}, v_{n}\right) c_{n} \sigma_{n} \cdots$ is consistent iff for all $i \geq 0: \gamma^{-1}\left(\ell_{i+1}, v_{i+1}\right)=\gamma^{-1}\left(\ell_{i}, v_{i}\right) \Longrightarrow c_{i+1}=c_{i}$. We note Play ${ }^{c o}(H)$ the set of consistent plays of $H$, and $\operatorname{Pref}^{c o}(H)$ the set of prefixes of consistent plays of $H$. Let $\rho^{H}=\left(l_{0}, v_{0}\right) c_{0} \sigma_{0} \cdots\left(l_{n-1}, v_{n-1}\right) c_{n-1} \sigma_{n-1}\left(l_{n}, v_{n}\right) \in$ $\operatorname{Pref}^{c o}(H) . \rho^{H}$ is a choice point if either $n=0$, or $n>0$ and $\gamma^{-1}\left(l_{n-1}, v_{n-1}\right) \neq$ $\gamma^{-1}\left(l_{n}, v_{n}\right)$. Note that we have that ChoicePoint $(H) \subseteq \operatorname{Pref}^{c o}(H) \subseteq \operatorname{Pref}(H)$ and $\operatorname{Play}^{c o}(H) \subseteq \operatorname{Play}(H)$.

Let $\rho^{H}=\left(l_{0}, v_{0}\right) c_{0} \sigma_{0} \cdots\left(l_{n}, v_{n}\right) c_{n} \sigma_{n} \cdots$ be a consistent play in $H$. Let $I=$ $\left\{m \mid \rho^{H}(m) \in\right.$ ChoicePoint $\left.(H)\right\}$. The stuttering free observation $\operatorname{Obs}\left(\rho^{H}\right)$ of $\rho^{H}$ is the sequence in $\left(\mathcal{O} . \Sigma_{1}\right)^{\omega}$ defined by:

- if $I=\left\{n_{0}, n_{1}, \cdots, n_{k}\right\}$ is finite,

$$
\operatorname{Obs}\left(\rho^{H}\right)=\gamma^{-1}\left(l_{n_{0}}, v_{n_{0}}\right) c_{n_{0}} \cdots \gamma^{-1}\left(l_{n_{k}}, v_{n_{k}}\right) c_{n_{k}}\left(\gamma^{-1}\left(l_{n_{k}}, v_{n_{k}}\right) c_{n_{k}}\right)^{\omega}
$$

- if $I=\left\{n_{0}, n_{1}, \cdots, n_{k}, \cdots\right\}$ is infinite,

$$
\operatorname{Obs}\left(\rho^{H}\right)=\gamma^{-1}\left(l_{n_{0}}, v_{n_{0}}\right) c_{n_{0}} \gamma^{-1}\left(l_{n_{1}}, v_{n_{1}}\right) c_{n_{1}} \cdots \gamma^{-1}\left(l_{n_{k}}, v_{n_{k}}\right) c_{n_{k}} \cdots
$$

We call it "stuttering free" as, for all $i \in I, \gamma^{-1}\left(l_{n_{i}}, v_{n_{i}}\right) \neq \gamma^{-1}\left(l_{n_{i+1}}, v_{n_{i+1}}\right)$ except when $I$ is finite, but in this case, only the last observation is repeated infinitely. Let $\rho^{H} \in$ ChoicePoint $(H)$, let $I=\left\{n_{0}, n_{1}, \cdots, n_{k}\right\}$ be the set of indices $n_{i}$ such that $\rho^{H}\left(n_{i}\right) \in$ ChoicePoint $(H)$. The (finite) observation of $\rho^{H}$, noted Obs ${ }^{*}\left(\rho^{H}\right)$, is $\gamma^{-1}\left(l_{n_{0}}, v_{n_{0}}\right) c_{n_{0}} \cdots \gamma^{-1}\left(l_{n_{k}}, v_{n_{k}}\right) c_{n_{k}} \gamma^{-1}\left(l_{n_{k}}, v_{n_{k}}\right) c_{n_{k}}$. We say that a strategy $\lambda^{H}$ is an observation based stuttering invariant (OBSI) strategy if the following property holds: for all $\rho_{1}^{H}, \rho_{2}^{H} \in \operatorname{Pref}^{c o}(H)$, let $n_{1}$ be the maximal value such that $\rho_{1}^{H}\left(n_{1}\right) \in$ ChoicePoint $(H)$, let $n_{2}$ be the maximal value such that $\rho_{2}^{H}\left(n_{2}\right) \in$ ChoicePoint $(H)$, if $\operatorname{Obs}^{*}\left(\rho_{1}^{H}\left(n_{1}\right)\right)=\operatorname{Obs}^{*}\left(\rho_{2}^{H}\left(n_{2}\right)\right)$ then $\lambda^{H}\left(\rho_{1}^{H}\right)=$ $\lambda^{H}\left(\rho_{2}^{H}\right)$.

Winning Conditions and Winning Strategies. Let $\rho^{H} \in \operatorname{Play}(H)$ s.t. $\operatorname{Obs}\left(\rho^{H}\right)=o_{0} c_{0} o_{1} c_{1} \ldots o_{n} c_{n} \ldots$ The projection $\operatorname{Obs}\left(\rho^{H}\right) \downarrow \mathcal{O}$ over $\mathcal{O}$ of $\operatorname{Obs}\left(\rho^{H}\right)$ is the sequence $o_{0} o_{1} \ldots o_{n} \ldots$ A winning condition $\mathcal{W}$ is a stuttering closed ${ }^{4}$ subset of $\mathcal{O}^{\omega}$. A strategy $\lambda^{H}$ for Player 1 is winning in $H$ for $\mathcal{W}$, if and only if, $\forall \rho \in$ Outcome $^{H}\left(\lambda^{H}\right) \cdot \operatorname{Obs}(\rho) \downarrow \mathcal{O} \in \mathcal{W}$.

To conclude this section, we define the control problem OBSI-CP we are interested in: let $H$ be a TGS with observations $\mathcal{O}, \mathcal{W}$ be a stuttering closed subset of $\mathcal{O}^{\omega}$,

$$
\text { is there an OBSI winning strategy in } H \text { for } \mathcal{W} \text { ? }
$$

\footnotetext{
${ }^{4}$ A language is stutter closed, if for any word $w$ in the language, the word $w^{\prime}$ obtained from $w$ by either adding a stuttering step (repeating a letter), or erasing a stuttering step, is also in the language.
} 
In case there is such a strategy, we would like to synthesize one. The problem of constructing a winning strategy is called the synthesis problem.

\section{Subset Construction for Timed Games}

In this section, we show how to transform a timed game of imperfect information into an equivalent game of perfect information. Let $H=\left(L, \iota, X, \delta, \Sigma_{1}, \Sigma_{2}\right.$, inv, $\mathcal{P})$ be an $M$-TGS and let $\mathcal{S}_{H}=\left(S, s_{0}, \Sigma_{1}, \Sigma_{2}, \rightarrow\right)$ be its semantics. In this section we assume delay $\in \Sigma_{1}$ but $H$ has no transition labeled delay.

Useful functions. Let $\sigma \in \Sigma_{1}$. We define the relation $\stackrel{\sigma}{\longrightarrow}_{n o b s}$ by: $(l, v) \stackrel{\sigma}{\rightarrow}_{n o b s}$ $\left(l^{\prime}, v^{\prime}\right)$ if there is a prefix $\rho=\left(\ell_{0}, v_{0}\right) c_{0} \sigma_{0}\left(\ell_{1}, v_{1}\right) c_{1} \sigma_{1} \cdots\left(l_{k}, v_{k}\right) c_{k} \sigma_{k}\left(\ell_{k+1}, v_{k+1}\right)$ in $\operatorname{Pref}((l, v), H)$ with $\left(\ell_{0}, v_{0}\right)=(l, v),\left(\ell_{k+1}, v_{k+1}\right)=\left(l^{\prime}, v^{\prime}\right), \forall 0 \leq i \leq k, c_{i}=\sigma$, $\gamma^{-1}\left(\ell_{i}, v_{i}\right)=\gamma^{-1}\left(\ell_{0}, v_{0}\right)$, and $\gamma^{-1}\left(\ell_{k+1}, v_{k+1}\right) \neq \gamma^{-1}\left(\ell_{0}, v_{0}\right)$. Notice that because of the definition of time transitions in Definition 3, if $\sigma_{i} \in \mathbb{R}_{>0}$ and $0 \leq i<k$ then $\gamma^{-1}\left(\ell_{i}, v_{i}\right)=\gamma^{-1}\left(\ell_{i+1}, v_{i+1}\right)$ and if $\sigma_{i} \in \mathbb{R}_{>0}$ and $i=k, \gamma^{-1}\left(\ell_{i}, v_{i}\right)=$ $\gamma^{-1}\left(\ell_{i}, v_{i}+t\right)$ for all $0 \leq t<\sigma_{i}, \gamma^{-1}\left(\ell_{i}, v_{i}\right) \neq \gamma^{-1}\left(\ell_{i}, v_{i}+t\right)$ and $\left(\ell_{i+1}, v_{i+1}\right)=$ $\left(\ell_{i}, v_{i}+\sigma_{i}\right)$ (i.e. $\sigma_{i}$ is the first instant at which the observation changes). By the constraints imposed by $\mathcal{B}(X, M)$ this first instant always exists. We define the function $\operatorname{Next}_{\sigma}(l, v)$ by:

$$
\operatorname{Next}_{\sigma}(l, v)=\left\{\left(l^{\prime}, v^{\prime}\right) \mid(l, v) \stackrel{\sigma}{\rightarrow} \text { nobs }\left(l^{\prime}, v^{\prime}\right)\right\}
$$

This function computes the first next states after $(l, v)$ which have an observation different from $\gamma^{-1}(l, v)$ when Player 1 continuously plays $\sigma$. Next is extended to sets of states as usual.

We also define the function $\operatorname{Sink}_{\sigma}(\cdot): L \times \mathbb{R}_{\geq 0}^{X} \rightarrow L \times \mathbb{R}_{\geq 0}^{X}$ for $\sigma \in \Sigma_{1}:\left(l^{\prime}, v^{\prime}\right) \in$ $\operatorname{Sink}_{\sigma}(l, v)$ iff there is an (infinite) play $^{5} \rho=\left(\ell_{0}, v_{0}\right) c_{0} \sigma_{0}\left(\ell_{1}, v_{1}\right) c_{1} \sigma_{1} \cdots\left(l_{k}, v_{k}\right)$ $c_{k} \sigma_{k}\left(\ell_{k+1}, v_{k+1}\right) \cdots$ in $\mathcal{S}_{H}$ such that: $\left(\ell_{0}, v_{0}\right)=(l, v),\left(\ell_{k+1}, v_{k+1}\right)=\left(l,{ }^{\prime} v^{\prime}\right)$, $\forall 0 \leq i, c_{i}=\sigma$, and $\forall 0 \leq i, \gamma^{-1}\left(l_{i}, v_{i}\right)=\gamma^{-1}\left(l_{0}, v_{0}\right)$.

Non-Deterministic Game (of Perfect Information). The games of perfect information that we consider here are (untimed) non-deterministic games, and they are defined as follows: in each state $s$ Player 1 chooses an action $\sigma$ and Player 2 chooses the next state among the $\sigma$-successors of $s$.

Definition 4 (Non-Deterministic Game). We define a non-deterministic game (NDG) to be a tuple $G=\left(S, \mu, \Sigma_{1}, \Delta, \mathcal{O}, \Gamma\right)$ where:

$-S=S_{0} \cup S_{1}$ is a set of states;

- $\mu \in S_{0}$ is the initial state of the game;

- $\Sigma_{1}$ is a finite alphabet modeling choices for Player 1;

$-\Delta \subseteq S_{0} \times \Sigma_{1} \times S$ is the transition relation;

\footnotetext{
${ }^{5}$ With an infinite number of discrete transitions because of the boundedness assumption. If needed we can add the requirement that this path is non-zeno if we want to rule out zeno-runs.
} 
$-\mathcal{O}$ is a finite set of observations;

$-\Gamma: \mathcal{O} \rightarrow 2^{S} \backslash \emptyset$ maps an observation to the set of states it represents, we assume $\Gamma$ partitions $S\left(\Gamma^{-1}(s)=o \Longleftrightarrow s \in \Gamma(o)\right)$.

Definition 5 (Plays in NDG). A play in $G$ from $s_{0}$ is either an infinite sequence $s_{0} a_{0} s_{1} a_{1} \ldots s_{n} a_{n} \ldots$ such that for all $i \geq 0, s_{i} \in S_{0}, a_{i} \in \Sigma_{1}$, and $\left(s_{i}, a_{i}, s_{n+1}\right) \in \Delta$ or a finite sequence $s_{0} a_{0} s_{1} a_{1} \ldots s_{n}$ such that all $i, 0 \leq$ $i<n, s_{i} \in S_{0},\left(s_{i}, a_{i}, s_{i+1}\right) \in \Delta$, and $s_{n} \in S_{1}$. We note $\operatorname{Play}\left(s_{0}, G\right)$ the set of plays starting in $s_{0}$ in $G$ and let $\operatorname{Play}(G)=\operatorname{Play}(\mu, G)$. The observation of an infinite play $\rho^{G}=s_{0} a_{0} s_{1} a_{1} \ldots s_{n} a_{n} \ldots$ is defined by $\operatorname{Obs}\left(\rho^{G}\right)=$ $\Gamma^{-1}\left(s_{0}\right) a_{0} \Gamma^{-1}\left(s_{1}\right) a_{1} \ldots \Gamma^{-1}\left(s_{n}\right) a_{n} \ldots$. If $\rho^{G}=s_{0} a_{0} s_{1} a_{1} s_{n-1} a_{n-1} \ldots s_{n} a_{n} s_{n+1}$ is finite then $\operatorname{Obs}\left(\rho^{G}\right)=\Gamma^{-1}\left(s_{0}\right) a_{0} \Gamma^{-1}\left(s_{1}\right) a_{1} \ldots \Gamma^{-1}\left(s_{n}\right) a_{n}\left(\Gamma^{-1}\left(s_{n+1}\right) a_{n}\right)^{\omega}$.

A prefix in $G$ is a finite sequence $s_{0} a_{0} s_{1} a_{1} \ldots s_{n}$ ending in $s_{n} \in S_{0}$, such that for all $i, 0 \leq i<n,\left(s_{i}, a_{i}, s_{i+1}\right) \in \Delta$. We let $\operatorname{Pref}(G)$ be the set of prefixes of $G$. The observation of a prefix is $\operatorname{Obs}\left(\rho^{G}\right)=\Gamma^{-1}\left(s_{0}\right) a_{0} \Gamma^{-1}\left(s_{1}\right) a_{1} \ldots a_{n} \Gamma^{-1}\left(s_{n}\right)$. For any $\rho^{G} \in \operatorname{Play}(G), \rho^{G}(n)=s_{0} a_{0} \cdots s_{n}$ is the prefix up to state $s_{n}$ and we let $\left|\rho^{G}\right|=n$ to be the length of $\rho^{G}$.

Remark 2. $S_{0}$ is the set of states where Player 1 has to make a choice of action. In $S_{1}$-states, Player 1 does not have any choice. A prefix ends in an $S_{0}$-state. Finite sequences ending in $S_{1}$-states are not prefixes but finite plays.

Strategies and Winning Conditions for NDG. A strategy in $G$ is a func$\operatorname{tion}^{6} \lambda^{G}: \operatorname{Pref}(G) \rightarrow \Sigma_{1}$. The outcome, Outcome ${ }^{G}\left(\lambda^{G}\right)$, of a strategy $\lambda^{G}$ is the set of (finite or infinite) plays $\rho^{G}=s_{0} a_{0} \cdots s_{n} a_{n} \cdots$ s.t. $s_{0}=\mu, \forall i \geq 0, a_{i}=$ $\lambda^{G}\left(\rho^{G}(i)\right)$. Let $\rho^{G}$ be a play of $G$. We let $\operatorname{Obs}\left(\rho^{G}\right) \downarrow \mathcal{O}$ be the projection of $\operatorname{Obs}\left(\rho^{G}\right)$ on $\mathcal{O}$. A winning condition $\mathcal{W}$ for $G$ is a subset of $\mathcal{O}^{\omega}$. A strategy $\lambda^{G}$ is winning for $\mathcal{W}$ in $G$ iff $\forall \rho^{G} \in \operatorname{Outcome}^{G}\left(\lambda^{G}\right), \operatorname{Obs}\left(\rho^{G}\right) \in \mathcal{W}$.

Remark 3. Strategies in NDG are based on the history of the game since the beginning: in this sense, this is a perfect information game.

\section{Knowledge Based Subset Construction.}

Definition 6. Given a game $H=\left(L, \iota, X, \delta, \Sigma_{1}, \Sigma_{2}\right.$, inv, $\left.\mathcal{O}, \gamma\right)$, we construct a $N D G G(H)=\left(S, \mu, \Sigma_{1}, \Delta, \mathcal{O}, \Gamma\right)$ as follows:

- let $\mathcal{V}=\left\{W \in 2^{L \times \mathbb{R}_{\geq 0}^{X}} \backslash \emptyset \mid \gamma^{-1}(l, v)=\gamma^{-1}\left(l^{\prime}, v^{\prime}\right)\right.$ for all $\left.(l, v),\left(l^{\prime}, v^{\prime}\right) \in W\right\}$,

$-S=\mathcal{V} \times\{0,1\}$, and we note $S_{0}$ the set $\mathcal{V} \times\{0\}$ and $S_{1}$ the set $\mathcal{V} \times\{1\}$,

$-\mu=(\{(\iota, \mathbf{0})\}, 0)$,

$-\Delta \subseteq S \times \Sigma_{1} \times S$ is the smallest relation that satisfies: $\left(\left(V_{1}, i\right), \sigma,\left(V_{2}, j\right)\right) \in \Delta$ if

- $i=0$. A consequence is that if $i=1$ (a state in $S_{1}$ ) there are no outgoing transitions.

\footnotetext{
${ }^{6}$ Notice that $S_{1}$-states have no outgoing transitions and we do not need to define a strategy for these states.
} 
- $j=0$ and $V_{2}=\operatorname{Next}_{\sigma}\left(V_{1}\right) \cap o$ for some $o \in \mathcal{O}$ such that Next $\operatorname{N}_{\sigma}\left(V_{1}\right) \cap o \neq \emptyset$, or

- $j=1$ if $\operatorname{Sink}_{\sigma}(s) \neq \emptyset$ for some $s \in V_{1}$ and $V_{2}=\cup_{s \in V_{1}} \operatorname{Sink}_{\sigma}(s)$,

$-\Gamma^{-1}: S \rightarrow \mathcal{O}$, and $\Gamma^{-1}((W, i))=\gamma^{-1}(v)$ for $v \in W$. Note that this is well-defined as $W$ is a set of states of $H$ that all share the same observation.

Notice that the game $G(H)$ is non-deterministic as there may be many transitions labeled by $\sigma$ and leaving a state $s$ to many different states with different observations. $G(H)$ is total for $S_{0}$ states: $\forall(V, 0) \in S_{0}, \forall \sigma \in \Sigma_{1}, \sigma \in \operatorname{Enabled}(V)$, because either there is an infinite path from some $s \in V$ with the same observation or there is an infinite path with a new observation (remember that time can elapse or Player 2 can do a discrete action from any state in $H$ ). Although non-deterministic $G(H)$ enjoys a weaker notion of determinism formalized by the following proposition:

Proposition 1. Let $(V, i)$ be a state of $G(H), \sigma \in \Sigma_{1}$ and $o \in \mathcal{O}$. There is at most one $\left(V^{\prime}, j\right)$ with $V^{\prime} \subseteq \gamma(o)$ s.t. $\left((V, i), \sigma,\left(V^{\prime}, j\right)\right) \in \Delta$.

Note also that if $\left((V, 0), \sigma,\left(V^{\prime}, 0\right)\right) \in \Delta$ then $\Gamma^{-1}((V, 0)) \neq \Gamma^{-1}\left(\left(V^{\prime}, 0\right)\right)$. We can relate the consistent plays in $H$ and plays in $G$. For that, we define the function Abs : $\operatorname{Play}^{c o}(H) \rightarrow \operatorname{Play}(G)$ as follows.

Definition 7 (Abs for Plays of $H$ ). Let $\rho^{H}=\left(l_{0}, v_{0}\right) c_{0} \sigma_{0}\left(l_{1}, v_{1}\right) \ldots\left(l_{m}, v_{m}\right)$ $c_{m} \sigma_{m} \ldots$ be in Play ${ }^{c o}(H)$. Let $I=\left\{j \in \mathbb{N} \mid \rho^{H}(j) \in\right.$ ChoicePoint $\left.(H)\right\}$. Then $\operatorname{Abs}\left(\rho^{H}\right)$ is defined by:

- if $I$ is a finite set, let $I=\left\{j_{0}, j_{1}, \cdots, j_{n}\right\}$. Define $\operatorname{Abs}\left(\rho^{H}\right)=s_{0} a_{0} s_{1} a_{1} \ldots s_{n}$ $a_{n} s_{n+1}$ by induction as follows:

1. $s_{0}=\left(\left\{\left(l_{0}, v_{0}\right)\right\}, 0\right), a_{0}=c_{j_{0}}$ (and $\left.j_{0}=0\right)$,

2. and for all $i, 0<i \leq n$, if $s_{i-1}=(V, 0)$ then let $V^{\prime}=\operatorname{Next}_{a_{i-1}}(V) \cap$ $\gamma^{-1}\left(v_{j_{i}}\right), s_{i}=\left(V^{\prime}, 0\right)$ and $a_{i}=c_{j_{i}}$.

3. As $\rho^{H}$ has a finite number of choice points, it must be the case that $\forall k \geq j_{n}, \gamma^{-1}\left(l_{k}, v_{k}\right)=\gamma^{-1}\left(l_{j_{n}}, v_{j_{n}}\right)$; moreover, because $\rho^{H}$ is consistent, $\forall k \geq j_{n}, c_{k}=c_{j_{n}}$. If $s_{n}=(V, 0)$ we let $V^{\prime}=\cup_{v \in V} \operatorname{Sink}_{c_{j_{n}}}(v)$. $V^{\prime}$ must be non empty and we define $s_{n+1}=\left(V^{\prime}, 1\right)$.

- if $I$ is an infinite set, let $I=\left\{j_{0}, j_{1}, \cdots, j_{n}, \cdots\right\}$. We define $\operatorname{Abs}\left(\rho^{H}\right)=$ $s_{0} a_{0} s_{1} a_{1} \ldots s_{n} a_{n} s_{n+1} \cdots$ by induction as follows:

1. $s_{0}=\left(\left\{\left(l_{0}, v_{0}\right)\right\}, 0\right), a_{0}=c_{j_{0}}$ (and $\left.j_{0}=0\right)$,

2. and for all $i \geq 1$, if $s_{i-1}=(V, 0)$ then let $V^{\prime}=\operatorname{Next}_{a_{i-1}}(V) \cap \gamma^{-1}\left(v_{j_{i}}\right)$, $s_{i}=\left(V^{\prime}, 0\right)$ and $a_{i}=c_{j_{i}}$.

Definition 8 (Abs for consistent prefixes). Let $\rho^{H}=\left(l_{0}, v_{0}\right) \ldots\left(l_{n-1}, v_{n-1}\right)$ $c_{n-1} \sigma_{n-1}\left(l_{n}, v_{n}\right) \in \operatorname{Pref}^{c o}(H)$ and, $I=\left\{j_{0}, j_{1}, \cdots, j_{m}\right\}$ be the set of choice points of $\rho^{H}$. Then $\operatorname{Abs}\left(\rho^{H}\right)=s_{0} a_{0} \ldots s_{m-1} a_{m-1} s_{m}$ with:

$-s_{0}=\left(\left\{\left(l_{0}, v_{0}\right)\right\}, 0\right)$,

- and for all $i, 0<i \leq m$, if $s_{i-1}=\left(V_{i-1}, 0\right)$ then $s_{i}=\left(V_{i}, 0\right)$ where $V_{i}=$ $\operatorname{Next}_{a_{i-1}}\left(V_{i-1}\right) \cap o_{i}$,

$-\forall 0 \leq i<m, a_{i}=c_{j_{i}}$. 
It can be checked that $\operatorname{Abs}\left(\rho^{H}\right)$ is well-defined for consistent plays as well for prefixes. The following theorem states the correctness of our construction:

Theorem 1. Let $\Phi$ be a stuttering closed subset of $\mathcal{O}^{\omega}$. Player 1 has a winning strategy in $G(H)$ for $\Phi$ iff Player 1 has an observation based stuttering invariant winning strategy in $H$ for $\Phi$.

\section{Symbolic Algorithms}

In this section, we show that the game $G(H)$ is a finite game and design an efficient symbolic algorithm for reachability and safety objectives.

Given a set of states $S$ represented as a finite union of zones, and an action $c \in$ $\Sigma_{1}$ we can compute the sets $\bigcup_{s \in S} \operatorname{Next}_{c}(s)$ and $\bigcup_{s \in S} \operatorname{Sink}_{c}(s)$ as finite unions of zones. Since the clocks are bounded in our $M$-TGS, only a finite number of zones are computed during the computation of those operators. As a consequence, the game $G(H)$ is finite.

To implement efficiently the computations, we use Difference Bound Matrices (DBMs), which allow efficient realisation of most set operations (inclusion, interesection, future, reset...) $[11,14]$.

Since $G(H)$ is finite, we can apply standard control algorithms to compute the set of winning states. In particular, for reachability or safety objectives, we can use the efficient on-the-fly forward algorithm of [9] that has been implemented in UPPAAL-Tiga [5].

The algorithm for finite games given in [9] can easily be tailored to solve NDGs of type $G=\left(\mathcal{S}, s_{0}, \Sigma_{1}, \Delta, \mathcal{O}, \Gamma\right)$ with reachability objective Goal s.t. Goal $\in \mathcal{O}$. Then, to obtain an algorithm for games of imperfect information, we replace the transition relation of $G(H)$ in this algorithm with the definition (see Definition 6) of the transition relation of $G(H)$ using the Next and Sink operators. This way we obtain the algorithm OTFPOR for TGS which is given Figure 2.

An important feature added to the algorithm is the propagation of losing state-sets, that is state-sets for which the reachability objective can be directly concluded not to be achievable. For reachability games, a state-set $W$ may declared to be losing provided it is not among the Goal sets and is a deadlock. Safety games are dual to reachability games in the sense that if the algorithm concludes that the initial state is not losing it is possible to extract a strategy to avoid losing states.

\section{$5 \quad$ Example and Experiments}

In this section we report on an application of a prototype implementation of the OTFPOR algorithm. Similar to the Box Painting Production System (BPPS) from the Introduction, we want to control a system consisting of a moving belt and an ejection piston at the end of the belt. However, a complicating feature compared with BPPS is that the system can receive both light and heavy boxes. 


\section{Initialization:}

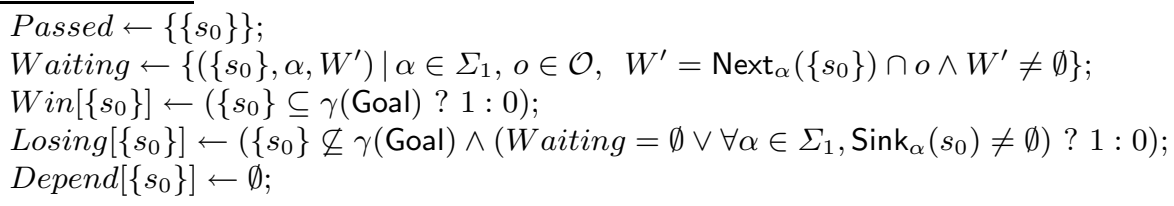

\section{Main:}

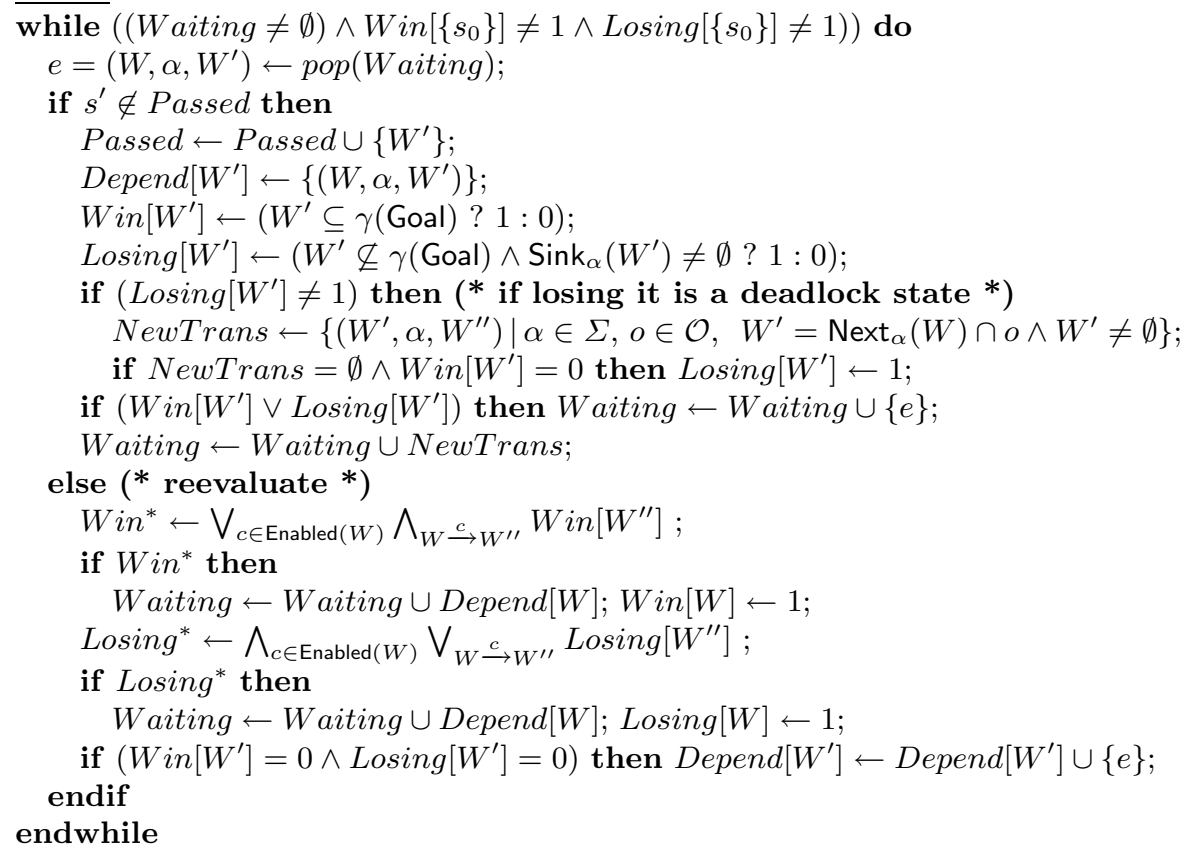

Fig. 2. OTFPOR: On-The-Fly Algorithm for Partially Observable Reachability

When receiving a light box, its speed is high and the box takes between 4 to 6 seconds to reach the zone where it can be Eject?'ed with a piston. When receiving a heavy box, the speed of the belt is slower and the box takes between 9 and 11 seconds to reach the zone where it can be Eject?'ed by the piston. The controller should command the piston so that the order to Eject? a box is given only when the box is in the right region. The system is modeled by the timed game automaton of Figure 3 . The initial location of the automaton is $l_{0}$. The system receives boxes when it is in location $l_{0}$, if it receives a heavy box then it branches to $l_{1}$, if it receives a light box then it branches to $l_{4}$. The only event that is shared with the controller is the Eject? event. This event should be issued when the control of the automaton is in $l_{3}$ or $l_{6}$ (which has the effect of ejecting the box at the end of the belt), in all other locations, if this event is received then the control of the automaton evolves to location $l_{7}$ (the bad location that 


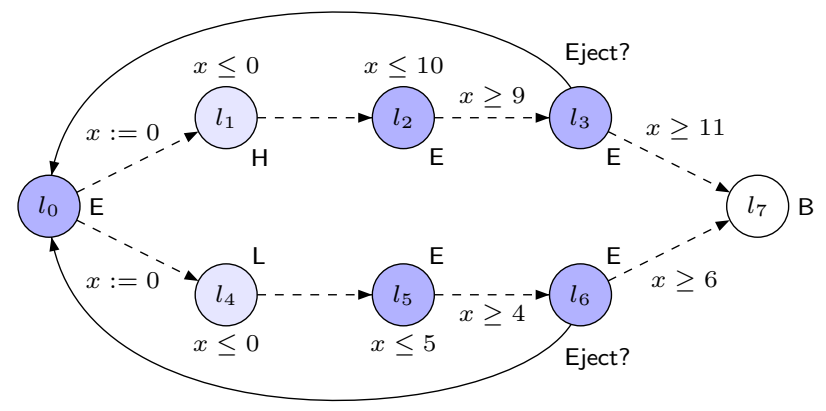

Fig. 3. Timed Game for Sorting Bricks. Edges to $l_{7}$ with action Eject? are omitted.

we want to avoid); those transitions are not depicted in the figure. The control objective is to avoid entering location $l_{7}$.

To control the system, the controller can use a clock $y$ that it can reset at any moment. The controller can also issue the order Eject! or propose to play delay, which allows for the time to elapse. The controller has an imperfect information about the state of the system and the value of the clock $y$. The controller gets information throughout the following observations: E: the control of the automaton is in location $l_{0}, l_{2}, l_{3}, l_{5}$, or $l_{6} ; \mathrm{H}$ : the control of the automaton is in location $l_{1}$; L: the control of the automaton is in location $l_{4}$; B: the control of the automaton is in location $l_{7} ; 0 \leq y<M$ : the value of clock $y$ is in the interval $[0, M[, M$ being a parameter. The observations $\mathrm{E}, \mathrm{H}, \mathrm{L}$, and $\mathrm{B}$ are mutually exclusive and cover all the states of the automaton but they can be combined with the observation $0 \leq y<M$ on the clock but also with its complement $y \geq M$. So formally, the set of observations that the controller can receive at any time is $\mathcal{O}=\{(\mathrm{E}, 0 \leq y<M),(\mathrm{E}, y \geq M),(\mathrm{H}, 0 \leq y<M),(\mathrm{H}, y \geq M),(\mathrm{L}, 0 \leq$ $y<M),(\mathrm{L}, y \geq M),(\mathrm{B}, 0 \leq y<M),(\mathrm{B}, y \geq M)\}$. The set of actions that the controller can choose from is $\Sigma_{c}=\left\{\right.$ Reset $_{y}$, Eject!, delay $\}$.

We modelled this example in our prototype and checked for controllability of the safety property $A \square \neg \mathrm{B}$. Controllability as well as the synthesized strategy heavily depend on the choice of the parameter $M$, i.e. the granularity at which the clock $y$ may be set and tested. Table 1 gives the experimental results for $M \in\{1,0.5,0.25,0.2\}^{7}$. It turns out that the system is not controllabe for $M=1$ : a granularity of 1 is simply too coarse to determine (up to that granularity) with certainty when, say, a light box will be in $l_{6}$ and should be Eject?'ed. The differences between the guards and invariants in $l_{5}, l_{6}$ and $l_{7}$ are simply too small. As can be seen from Table 1 the finer granularities yield controllability.

We report on the number of explored state-sets (state-set) and the number of state-sets that are part of the strategy (strat). To get an impression of the complexity of the problem of controller synthesis under partial observability we note that the the model in Figure 3 has 115 reachable symbolic states when viewed

\footnotetext{
${ }^{7}$ Fractional values of $M$ are dealt with by multiplying all constants in the timed game automaton with $\frac{1}{M}$.
} 


\begin{tabular}{|c|c|c|c|c|c|c|c|c|}
\hline & \multicolumn{4}{|c|}{ notfi } & \multicolumn{4}{|c|}{ otfi } \\
\hline & state-set & strat & $1+$ post & |+filter & state-set & strat & $1+$ post & +filter \\
\hline$[0,0.2[$ & 110953 & 36169 & 1244 & 70 & 52615 & 16372 & 841 & 176 \\
\hline$[0,0.25$ & 72829 & 23750 & 1014 & 60 & 35050 & 11016 & 697 & 146 \\
\hline$[0,0.5[$ & 20527 & 6706 & 561 & 41 & 10586 & 3460 & 407 & 88 \\
\hline$[0,1[$ & 2284 & - & - & - & 1651 & - & - & - \\
\hline
\end{tabular}

Table 1. Number of state-sets and size of strategy obtained for different heuristics for observations $0 \leq y<M$ of the clock $y$ with $M \in\{1,0.5,0.25,0.2\}$. The case $M=1$ is not controllable.

as a regular timed automaton. Table 1 reports on experiments exploiting an additional inclusion checking option in various ways: (notfi) without on-the-fly inclusion checking, and (otfi) with on-the-fly inclusion checking. In addition, we apply the post-processing step of inclusion checking $(+$ post $)$ on the strategy and a filtering of the strategy $(+$ filter $)$ on top to output only the reachable state-sets under the identified strategy. The results show that on-the-fly inclusion checking gives a substantial reduction in the number of explored state-sets - and is hence substantially faster. Both (notfi) and (otfi) shows that post processing and filtering reduces the size of the control strategy with a factor of approximately 100 . It can also be seen that the size of the final strategy grows when granularity is refined; this is to be expected as the strategies synthesized can be seen to involve counting modulo the given granularity. More suprising is the observation that the final strategies in $(n o t f i+$ post + filter $)$ are uniformly smaller than the final strategies in (otfi): not performing on-the-fly inclusion checking explores more state-sets, thus having the potential for a better reduction overall.

\section{Conclusions and Future Works}

During the last five years a number of very promissing algorithmic techniques has been introduced for controller synthesis in general and controller synthesis for timed systems in particular. The contribution of this paper to the decidability and algorithmic support for timed controller synthesis under imperfect information is an important new step within this line of research. Future research includes more experimental investigation as well as search for additional techniques for minimizing the size of the produced strategies (e.g. using minimization wrt. (bi)simulation or alternating simulation). For safety objectives, we need methods to insure that the synthesized strategies do not obtain their objective simply by introducing zeno behaviour. Finally, a rewrite of the prototype as extension of UPPAAL-TigA is planned.

\section{References}

1. K. Altisen and S. Tripakis. Tools for controller synthesis of timed systems. In Proc. 2nd Work. on Real-Time Tools (RT-TOOLS'02), 2002. Proc. published as 
Technical Report 2002-025, Uppsala University, Sweden.

2. R. Alur and D. Dill. A theory of timed automata. Theoretical Computer Science, 126(2):183-235, 1994.

3. E. Asarin and O. Maler. As Soon as Possible: Time Optimal Control for Timed Automata. In Proc. 2nd Work. Hybrid Systems: Computation \& Control (HSCC'99), volume 1569 of LNCS, pages 19-30. Springer, 1999.

4. E. Asarin, O. Maler, A. Pnueli, and J. Sifakis. Controller Synthesis for Timed Automata. In Proc. IFAC Symp. on System Structure 8 Control, pages 469-474. Elsevier Science, 1998.

5. G. Behrmann, A. Cougnard, A. David, E. Fleury, K. Larsen, and D. Lime. Uppaaltiga: Time for playing games! In Proc. of 19th Int. Conf. on Computer Aided Verification (CAV'07), volume 4590 of Lecture Notes in Computer Science, pages 121-125, Berlin, Germany, 2007. Springer.

6. G. Behrmann, A. David, and K. G. Larsen. A tutorial on uppaal. In M. Bernardo and F. Corradini, editors, SFM, volume 3185 of Lecture Notes in Computer Science, pages 200-236. Springer, 2004.

7. G. Behrmann, A. David, K. G. Larsen, J. Håkansson, P. Pettersson, W. Yi, and M. Hendriks. Uppaal 4.0. In QEST, pages 125-126. IEEE Computer Society, 2006.

8. P. Bouyer, D. D'Souza, P. Madhusudan, and A. Petit. Timed control with partial observability. In W. A. Hunt, Jr and F. Somenzi, editors, Proc. of the 15th Int. Conf. on Computer Aided Verification (CAV'03), volume 2725 of Lecture Notes in Computer Science, pages 180-192, Boulder, Colorado, USA, July 2003. Springer.

9. F. Cassez, A. David, E. Fleury, K. Larsen, and D. Lime. Efficient on-the-fly algorithms for the analysis of timed games. In M. Abadi and L. de Alfaro, editors, Proc. of the 16th Int. Conf. on Concurrency Theory (CONCUR'05), vol. 3653 of Lecture Notes in Computer Science, pp 66-80, San Francisco, CA, USA, 2005. Springer.

10. K. Chatterjee, L. Doyen, T. A. Henzinger, and J.-F. Raskin. Algorithms for omegaregular games with imperfect information' . In Z. Ésik, editor, CSL, volume 4207 of Lecture Notes in Computer Science, pages 287-302. Springer, 2006.

11. D. Dill. Timing Assumptions and Verification of Finite-State Concurrent Systems. In Workshop on Automatic Verification Methods for Finite-State Systems, volume 407, pages 197-212, 1989.

12. F. Laroussinie and K. G. Larsen. CMC: A tool for compositional model-checking of real-time systems. In S. Budkowski, A. R. Cavalli, and E. Najm, editors, Proc. of IFIP TC6 WG6.1 Joint Int. Conf. FORTE'XI and PSTV'XVIII, volume 135 of IFIP Conf. Proc., pages 439-456, Paris, France, Nov. 1998. Kluwer Academic Publishers.

13. F. Laroussinie, K. G. Larsen, and C. Weise. From timed automata to logic - and back. In Proc. $20^{\text {th }}$ Symp. on Mathematical Foundations of Computer Science (MFCS'95), volume 969 of LNCS, pages 529-539. Springer, 1995.

14. K. Larsen, P. Pettersson, and W. Yi. Model-checking for real-time systems. In Fundamentals of Computation Theory, pages 62-88, 1995.

15. O. Maler, A. Pnueli, and J. Sifakis. On the synthesis of discrete controllers for timed systems. In Proc. 12 ${ }^{\text {th }}$ Symp. on Theoretical Aspects of Computer Science (STACS'95), volume 900, pages 229-242. Springer, 1995.

16. S. Tripakis and K. Altisen. On-the-Fly Controller Synthesis for Discrete and Timed Systems. In Proc. of World Congress on Formal Methods (FM'99), volume 1708 of $L N C S$, pages 233-252. Springer, 1999.

17. M. D. Wulf, L. Doyen, and J.-F. Raskin. A lattice theory for solving games of imperfect information. In J. P. Hespanha and A. Tiwari, editors, $H S C C$, volume 3927 of Lecture Notes in Computer Science, pages 153-168. Springer, 2006. 